\title{
Reliability and repeatability of testing visual evoked potential habituation in migraine: A blinded case-control study
}

Cephalalgia

$0(0) \mathrm{I}-5$

(C) International Headache Society 2016 Reprints and permissions: sagepub.co.uk/journalsPermissions.nav DOI: $10.1177 / 0333102416648656$ cep.sagepub.com

@SAGE

\author{
Anna Ambrosini', Gianluca Coppola ${ }^{2}$, Ennio lezzi ${ }^{3}$, \\ Francesco Pierelli, ${ }^{1,4}$ and Jean Schoenen ${ }^{5}$
}

\begin{abstract}
Background: Many studies have shown that migraine patients have an interictal habituation deficit of visual evoked potentials (VEPs). Some discordant results were attributed to non-blinded analyses and a lack of repeatability.

Aims: In this study, we compared blinded and non-blinded analyses of the same recordings and assessed test-retest repeatability.

Methods: VEP recordings of 25 healthy volunteers (HVs) and 78 episodic migraine patients (EMs; 52 interictal, 26 ictal) were analysed by two investigators, one of whom was blinded to diagnosis and headache phase. Twelve HVs and nine EMs had two recordings for test repeatability.

Results: In both blinded and non-blinded analyses, VEP habituation was normal in HVs and EMs during an attack, but deficient in EMs interictally. Intra-individual habituation percentages were highly correlated in two recordings separated by $\geq 7$ days.

Conclusions: The studies showing a VEP habituation deficit in migraineurs between attacks are unlikely to be biased by non-blinding analysis or poor repeatability.
\end{abstract}

\section{Keywords}

Migraine, visual evoked potentials, habituation

Date received: 27 November 2015; revised: 14 March 2016; accepted: 16 March 2016

\section{Introduction}

Many studies have suggested that migraine patients are characterised between attacks by a habituation deficit of visual evoked potentials (VEPs) (1). Some researchers, however, have been unable to reproduce these findings $(2-5)$. This was tentatively attributed to methodological issues, such as non-blinded analyses $(4,5)$, although four VEP studies (6-9) in which investigators were kept unaware of the diagnosis found the same significant abnormality between migraine patients and healthy controls. Another possible bias could be poor test repeatability, which would increase the variance in groups of subjects. In a more recent VEP study that confirmed the habituation deficit in migraine patients, repeatability over time was not found to be sufficient to allow for reliable comparisons in the same patient (10).

We therefore decided to compare blinded and nonblinded measures of VEP habituation and their reproducibility in repeated recordings.

\section{Methods}

\section{Subjects}

Seventy-eight episodic migraine patients (EMs) were recruited at the Headache Clinic of the University of Rome, Polo Pontino (Latina, Italy - Centre 1) and at the Headache Clinic of the IRCCS Neuromed

\footnotetext{
'Headache Unit, IRCCS Neuromed, Pozzilli (Isernia), Italy

${ }^{2}$ Department of Neurophysiology of Vision and Neurophthalmology, G.B. Bietti Foundation IRCCS, Rome, Italy

${ }^{3}$ Clinical Neurophysiology Unit, IRCCS Neuromed, Pozzilli (Isernia), Italy

${ }^{4}$ Sapienza University of Rome Polo Pontino, Department of Medico-

Surgical Sciences and Biotechnologies, Latina, Italy

${ }^{5}$ Headache Research Unit, Citadelle Hospital, University of Liège, Belgium
}

\section{Corresponding author:}

Anna Ambrosini, Department of Headache Unit, IRCCS Neuromed, Via Atinense, 18, I 86077, Pozzilli (Isernia), Italy.

Email: anna.ambrosini@neuromed.it 
(Pozzilli, Italy - Centre 2). Migraineurs and 25 healthy volunteers (HVs) of comparable age and gender distributions underwent electrophysiological recordings at the same sites. We excluded subjects with uncorrected visual deficits or drug intake on a regular basis or within 3 days of the recordings. In migraine patients, the recordings were performed at least 3 days after or before an attack, with the occurrence of an attack after the recordings being verified by a phone call. Some migraine patients in Centre 1 also had VEP recordings during an attack. Local Ethics Committees approved the study and, in accordance with the ethical standards of the 1964 Declaration of Helsinki, informed consent was obtained from each subject.

\section{Recordings}

VEP recordings and analyses. In Centre 1, electroencephalogram (EEG) signals were amplified with a Digitimer D360 amplifier (band-pass $0.05-2000 \mathrm{~Hz}$, gain 1000) and recorded with a $\mathrm{CED}^{\mathrm{TM}}$ power 1401 device (Cambridge Electronic Design Ltd, Cambridge, UK). Responses in each block were averaged offline using the Signal ${ }^{\mathrm{TM}}$ software package version 4.10 (Cambridge Electronic Design Ltd). In Centre 2, signals were recorded and averaged online with a Medelec Synergy, PLINTH, CareFusion (Middleton, WI, USA) device. Subjects were comfortably seated in an armchair with one eye patched in front of a television monitor placed at $100 \mathrm{~cm}$ in a quiet room with a surrounding luminance of $5 \mathrm{~cd} / \mathrm{m}^{2}$. After adaptation for at least 10 minutes, a checkerboard pattern of black and white squares subtending 15' of arc (contrast $80 \%$ ) was presented at a reversal frequency of $3.1 \mathrm{~Hz}$ while subjects were instructed to fixate on a red dot in the middle of a screen. EEG signals were recorded with scalp-inserted needle electrodes at $\mathrm{Oz}$ (active electrode) and $\mathrm{Fz}$ (reference) according to the 10-20 EEG reference system, with a superficial adhesive ground electrode placed on the right forearm. Six sequential blocks of 100 sweeps were collected over a total stimulation time of 3 minutes 20 seconds and analysed (VEP $100 \times 6$ ). The N1 component was identified as the most negative point at between 60 and $90 \mathrm{~ms}$ after the stimulus and the P1 component as the most positive point at between 80 and $120 \mathrm{~ms}$. In each block, we measured N1 and P1 latencies and peak-topeak N1-P1 amplitudes. In order to assess habituation, the change in N1-P1 amplitude between the sixth and the first block was expressed in percentages (negative values = habituation; positive values $=$ potentiation).

Comparison of blinded and non-blinded VEP analyses. VEP $100 \times 6$ recordings were compared in 25 HVs (mean age $27.5 \pm 8.1$ years, 11 males/14 females) and 78 EMs. Fifty-two migraine patients (30 without aura (MO): mean age $29.5 \pm 8.0$ years, 5 males/ 25 females; 22 with aura: mean age $29.5 \pm 9.0$ years; 3 males $/ 19$ females) had interictal recordings, whereas 26 patients (MO: mean age $32.6 \pm 11.5$ years, 7 males/19 females) were studied during an attack. The recordings were analysed separately by two investigators (AA and GC), one of whom (AA) was blinded to the subjects and migraine phase. Both of these investigators independently performed an offline analysis of raw single trial data of the N1-P1 VEP component, including rejection of artefacts, averaging of trial blocks and calculation of habituation percentages.

Repeatability of VEP data. In Centre 2, a subgroup of subjects, $12 \mathrm{HVs}$ (mean age $31.6 \pm 7.7$ years; 6 males $/ 6$ females) and nine EMs (MO: mean age $35.1 \pm 10.4$ years, 4 males $/ 5$ females), underwent two VEP $100 \times 6$ recordings separated by a time interval ranging from 7 to 120 days (mean $60 \pm 38$ days). The recordings were performed according to the methods described above and analysed by an investigator (EI) who was blinded to the diagnosis.

\section{Statistical analyses}

The Shapiro-Wilk test was used to test the normality of the distributions. The results were expressed as means and standard deviations. The significance level was set at $p \leq 0.05$.

The results obtained by the blinded and the nonblinded investigators were compared separately by one-way analysis of variance with a post-hoc least significant difference (LSD) test. Intra-individual results were analysed with paired Student's $t$-tests, while correlations between blinded or non-blinded analyses and in the test-retest analysis were assessed with the intraclass correlation coefficient test (ICC) (two-way mixed, average measures, absolute agreement). Statistical analyses were performed with IBM SPSS Statistics for Windows, version 21.0 (IBM Corp., Armonk, NY, USA).

\section{Results}

The results are summarized in Table 1 .

\section{Blinded versus non-blinded VEP analyses}

In both the blinded and the non-blinded analyses, VEP habituation was present in HVs, but deficient in interictal EMs. The difference between HVs and EMs was significant both for the blinded $(p=0.038)$ and the nonblinded protocols $(p<0.001)$. During an attack, habituation was normal in EM patients whether analysed 


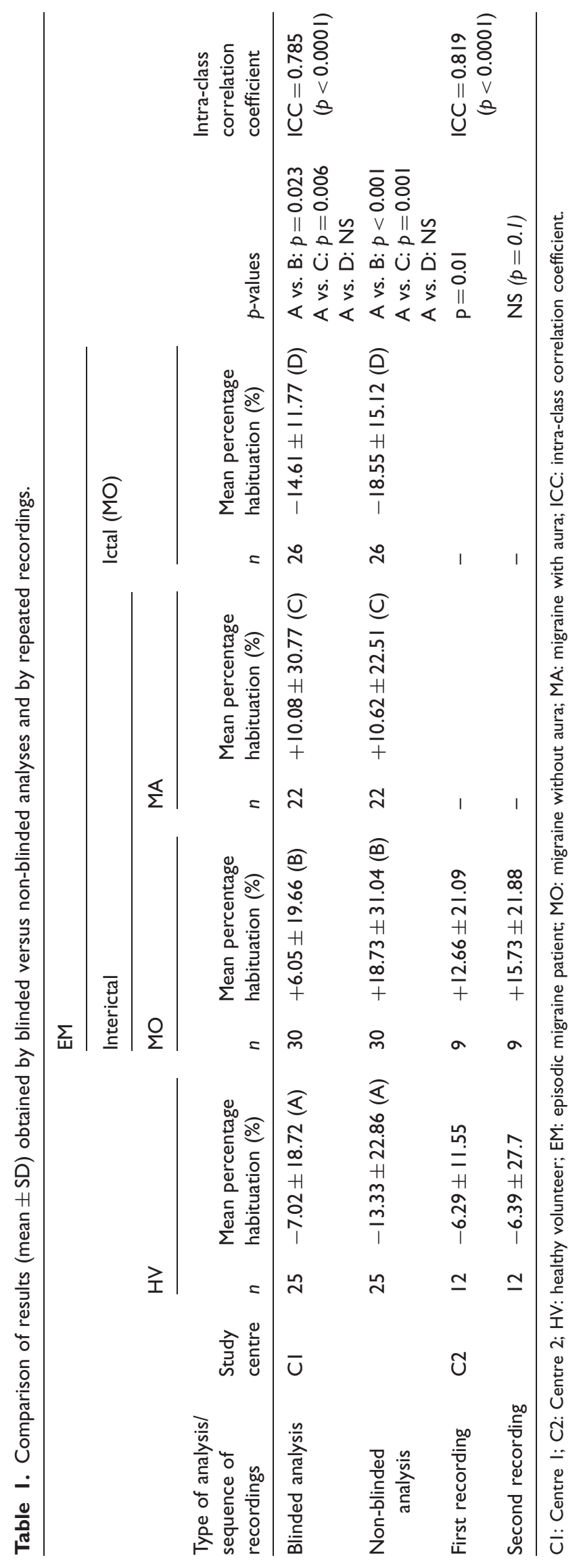


blindly or non-blindly. Intra-individual habituation percentages for both procedures were correlated in the pooled HV and EM study groups $(\mathrm{ICC}=0.785)$.

\section{Repeatability of VEP results}

In the $12 \mathrm{HVs}$ and 9 EMs in whom VEP $100 \times 6$ recordings were repeated twice, habituation values and differences between study groups were similar in the first and second recordings. Intra-individual data for both tests were significantly correlated $(\mathrm{ICC}=0.819)$ in the total group of 21 subjects.

\section{Discussion}

Our data show that habituation of VEPs is on average deficient in episodic migraine patients between attacks, whether the recordings are analysed blindly or not. This is in line with four blinded VEP studies (6-9) and suggests that the differences between the studies that found a deficit of habituation in migraineurs $(1,6-10)$ and those which reported normal habituation (2-5) cannot be explained by the blinding or not of the analyses.

Moreover, we confirmed in a blinded analysis of a subgroup of migraineurs our previous finding $(7,11)$ that VEP habituation is within normal values when the recordings are performed during an attack. In another study (10), test-retest reliability of VEP habituation was poor (ICC $=-0.06$ for percentage habituation; $\mathrm{ICC}=0.30$ for habituation slope), although the authors confirmed that habituation was significantly decreased in migraine patients compared to healthy controls. This difference could in part be due to the fact that the time limit for excluding subjects with possible ictal/ peri-ictal changes was less stringent in Rauschel et al.'s (10) study ( \pm 48 hours compared to \pm 72 hours in our recordings). Moreover, the test-retest time interval between the two VEP recordings was either 2-3 weeks or 15 minutes in Rauschel et al.'s study (10), while it was on average 60 days in ours. Future test-retest studies could preferably use the binary criterion of 'normal or abnormal' according to defined thresholds (12), instead of considering more variable absolute habituation values. In our study, 17 subjects out of 21 had the same normal/abnormal habituation rating at retest. Thus, insufficient repeatability might play a minor role in the discrepancy of the results obtained in different centres for VEP habituation in migraine patients.
Finally, certain limitations of the present study should be acknowledged. First, the protocol could have been optimised if different investigators with expertise in VEP recordings, but unaware of the study aim and naïve to the matter of VEP habituation in migraine, had performed both blinded and non-blinded VEP analyses. Unconscious bias does not seem to have played a major role, however, since a study using automatic offline VEP analyses with a peak identification software tool (i.e. without intervention of the investigators) also found a habituation deficit in migraine (6). Second, in our study, the investigators were blinded during offline analyses of VEP data, but not during the recording sessions, although this probably only represents a minor risk for bias. Non-blinding to diagnosis probably does not explain the discrepancies between studies. In fact, even in VEP studies that were claimed to be blinded to diagnosis $(4,5,13)$, the reported methodology suggests that blinding was not perfect for various reasons (see discussion in (9)), which might have biased the expectations of the examiners (14). In a clinical setting, it thus seems quasi-impossible to totally blind a neurophysiological study (see discussion in (9)). Finally, the notion that blinding may play a minor role in the reported results is suggested by the fact that the authors, who found normal VEP habituation in migraine patients, did so whether their study was blinded or not (3).

Other factors could be more relevant to the differences between studies. The neurophysiological phenotypes may depend on underlying genotypes that differ between patients coming from distant geographical areas. In another study (12), we indeed found a trend for a greater VEP habituation deficit in Italian than in Belgian migraine patients. If this reflects a difference in daylight illumination or, more likely, a different genetic background, one may hypothesise that there could be a negative gradient for the VEP habituation deficit between southern and northern European populations. As a matter of fact, we have previously identified two different neurophysiological phenotypes in a study in which the intensity dependence of auditory cortical potentials, which is heavily influenced by cortical habituation (15), was sensitised by simultaneous high-frequency flash stimulation (16). Studies of genotype-phenotype correlations and means of sensitising electrophysiological tests for the detection of more subtle abnormalities are necessary in order to verify this hypothesis. 


\section{Article highlights}

- In this study, we confirm that migraine patients have an interictal deficit of habituation to repeated visual stimuli.

- This deficit can also be demonstrated when visual evoked potential recordings are analysed blindly and recordings repeated in separated sessions. We found no significant difference in individual habituation values between the blinded and the non-blinded analyses and in individual repeated recordings.

- The discrepant findings in the literature thus most likely cannot be explained by the presence or absence of blinding, nor by poor repeatability.

\section{Declaration of conflicting interests}

The authors declared no potential conflicts of interest with respect to the research, authorship and/or publication of this article.

\section{Funding}

The authors disclosed receipt of the following financial support for the research, authorship, and/or publication of this article: JS was supported by research convention 3.4.650.09 of the National Fund for Scientific Research - Belgium, Special Research Funds and grants from the Fonds Frédéricq of the Faculty of Medicine -University of Liège and in part by FP7EUROHEADPAIN project no. 602633; GC was financially supported by the Italian Ministry of Health and Fondazione Roma.

\section{References}

1. de Tommaso M, Ambrosini A, Brighina F, et al. Altered processing of sensory stimuli in patients with migraine. Nat Rev Neurol 2014; 10: 144-155.

2. Oelkers R, Grosser K, Lang E, et al. Visual evoked potentials in migraine patients: alterations depend on pattern spatial frequency. Brain 1999; 122: 1147-1155.

3. Sand T and Vingen JV. Visual, long-latency auditory and brainstem auditory evoked potentials in migraine: relation to pattern size, stimulus intensity, sound and light discomfort thresholds and pre-attack state. Cephalalgia 2000; 20: 804-820.

4. Omland PM, Nilsen KB, Uglem M, et al. Visual evoked potentials in interictal migraine: no confirmation of abnormal habituation. Headache 2013; 53: 1071-1086.

5. Omland PM, Uglem M, Hagen K, et al. Visual evoked potentials in migraine: is the "neurophysiological hallmark" concept still valid? Clin Neurophysiol 2016; 127: $810-816$

6. Bednář M, Kubová Z and Kremláček J. Lack of visual evoked potentials amplitude decrement during prolonged reversal and motion stimulation in migraineurs. Clin Neurophysiol 2014; 125: 1223-1230.
7. Coppola G, Parisi V, Di Lorenzo C, et al. Lateral inhibition in visual cortex of migraine patients between attacks. J Headache Pain 2013; 14: 20.

8. Ambrosini A, Iezzi E, Perrotta A, et al. Correlation between habituation of visual-evoked potentials and magnetophosphene thresholds in migraine: a case-control study. Cephalalgia 2016; 36: 258-264.

9. Coppola G, Bracaglia M, Di Lenola D, et al. Visual evoked potentials in subgroups of migraine with aura patients. J Headache Pain 2015; 16: 92.

10. Rauschel V, Ruscheweyh R, Krafczyk S, et al. Test-retest reliability of visual-evoked potential habituation. Cephalalgia. Epub ahead of print 23 October 2015. DOI: $10.1177 / 0333102415613613$.

11. Judit A, Sándor PS and Schoenen J. Habituation of visual and intensity dependence of auditory evoked cortical potentials tends to normalize just before and during the migraine attack. Cephalalgia 2000; 20: 714-719.

12. Ambrosini A, Kisialiou A, Finos L, et al. The diagnostic value of visual and auditory evoked potentials in migraine: a retrospective multicenter study. Cephalalgia 2013; 33(Suppl. 8): 153-154.

13. Omland PM, Uglem M, Engstrøm M, et al. Modulation of visual evoked potentials by high-frequency repetitive transcranial magnetic stimulation in migraineurs. Clin Neurophysiol 2014; 125: 2090-2099.

14. Brighina F, Cosentino $G$ and Fierro B. Habituation or lack of habituation: what is really lacking in migraine? Clin Neurophysiol 2016; 127: 19-20.

15. Ambrosini A, Rossi P, De Pasqua V, et al. Lack of habituation causes high intensity dependence of auditory evoked cortical potentials in migraine. Brain 2003; 126 : 2009-2015.

16. Ambrosini A, Coppola G, Gérardy PY, et al. Intensity dependence of auditory evoked potentials during light interference in migraine. Neurosci Lett 2011; 492: 80-83. 